Original Research Paper

\title{
Asymptotic Stability of Unicycle-Like Robots: The Bessel's Controller
}

\author{
Andrés G. García \\ Grupo de Investigación de Multifísica Aplicada (GIMAP), Universidad Tecnológica Nacional, \\ Facultad Regional Bahía Blanca, 11 de Abril 461, Bahía Blanca, Buenos Aires, Argentina
}

Article history

Received: 29-11-2019

Revised: 13-01-2020

Accepted: 16-01-2020

Email: andresgarcia@frbb.utn.edu.ar

\begin{abstract}
Asymptotic stability of unicycle-like robots proved to be involved due to Brockett's condition. By using a smooth, time-invariant controller constructed out of Bessel's functions, in this paper unicycle-like robots are uniformly-exponentially stabilized to the origin. The pure feedback controller obtained provides closed-form trajectories with the possibility of a simple and feasible (hardware) non-linear observer construction from posture angle measurements solely. Two examples are presented: Asymptotic steering of a unicycle to the origin using gyros and a perfect non-linear observer reconstruction states along with conclusions and future work.
\end{abstract}

Keywords: Nonholonomic Dynamics, Brockett's Condition, Kinematic Model, Closed-Loop, Closed-Form Solution

\section{Introduction}

Modelling mechanical systems can be carried out in two main ways (Angeles and Kecskemethy, 1995):

- Dynamic models including forces and torques

- Kinematic models excluding forces and torques

Both approaches aim to collect a system of Ordinary Differential Equations (ODE's) parameterized in its control inputs (Astolfi et al., 1997; Bloch, 2015).

These ODE systems are mainly non-linear, with a universal modeling given by Sarkar et al. (1994) in the case of kinematic rolling constraints.

It turns out that dynamic models represent the most general approach to account for all possible physical interactions that may occur. However, these models are out of as many ODE's as the mechanical systems' degrees of freedom (Kane and Levinson, 1985; Angeles and Kecskemethy, 1995).

At this point, two main challenges must be dealed with:

- A great amount of ODE's

- A control law rendering the system asymptotically stable/stable (Kostić et al., 2009; Udwadia and Kalaba, 1994; Skowronski, 2012)

For these reasons, many researchers focus the attention on more tractable models, yet keeping the nonlinear richness with fewer amounts of ODE's (MuñozLecanda and Yániz Fernandez, 2008).
This explains the great interest in controlling kinematic models (Siegwart et al., 2011) with the case of mobile robots as a subset of kinematic modeling, mainly, wheel planar kinematic models.

Moreover, it happens that these models can be classified into two universal classes: Holonomic and Nonholonomic (Garcia and Agamennoni (2012) for a universal classification and models).

As pointed out by Jian et al. (2010), El-Hawwary and Maggiore (2008) and $\mathrm{Qu}$ et al. (2004), holonomic robots are simpler than nonholonomic, being one of the reasons that many techniques have been proposed without any convergence's guarantee (Lavalle, 2006; Galceran and Carreras, 2013; Kumar and Dewangan, 2016; Yang et al., 2016; Salaris et al., 2010; Wang et al., 2009; Thomas et al., 2016).

On the other hand, it is well-known that a nonholonmic robot cannot be stabilized asymptotically with a smooth controller due to Brockett's condition (Brockett, 1983).

For this reason, many different techniques have been proposed to control nonholonomic robots avoiding the use of time-invariant controllers (Zambelli et al. (2015) and the references therein).

However, none of the available techniques considers the stabilization's problem in closed-form. In fact, according to Lizárraga (2004), it is not possible to track some desired trajectories with an equi-continuous control law.

To summarize the literature's drawbacks in controlling nonholonomic robots, either path-following or asymptotic stability: 
- Planar curves must be parameterized to be followed $f(x, y)=0$ (Morro et al. (2011))

- Path's curvature must satisfy some specifications: $\nabla f(x, y) \neq 0$ ( Morro et al. (2011))

- Very oscillatory and slow motion (see for instance Moon Kim and Tsiotras (2002))

- Brockett's condition

- Lizárraga's obstructions

- Closed-form solution's unavailability for a universal set of models

- Closed-form algorithms to track/follow any desired pre-specified trajectory

In this paper, generalizing the solution presented in Garcia et al. (2008), a smooth time-invariant controller is presented to steer in closed-loop a unicycle-like robot to the origin uniformly exponentially stable.

The contributions in this paper are as follows:

- Closed-form solution to steer unicycle robots to the origin

- Continuous feedback controller with guaranteed stability

- The proof that a unicycle can be asymptotically stabilized measuring considering modularity

- A non-linear observer with angle output measurement (gyros)

This paper is organized as follows: Section Rolling constraints presents the modeling of rolling constraints to be considered, trajectories' closed-form solution using a smooth, time-invariant controller, Section Unicycle's asymptotic stability presents the asymptotic stability analysis, Section Practical controller: Only gyros presents a practical algorithm, whereas Section Examples simulates in Matlab. Finally, Section Conclusions depicts some conclusions and future work.

\section{Notations and Definitions}

In this short section, some definitions are provided to use all along the paper:

\section{Rotation Matrix}

$$
R(x)=\left[\begin{array}{cc}
\cos (x) & \sin (x) \\
-\sin (x) & \cos (x)
\end{array}\right]
$$

\section{Matrix Eigenvalues}

Given a matrix $A \in \mathfrak{R}^{n \times n}$, the eigenvalues $\lambda(A)$ :

$$
\lambda(A)=\operatorname{det}(\lambda \cdot I-A)=0
$$

With $I$ the identity matrix.

\section{Matrix Transpose}

Given a matrix $A \in \mathfrak{R}^{n \times n}$, the transpose is denoted by $A^{\prime}$.

\section{Derivatives with Respect to Time}

Time derivatives are indicated as:

$$
\dot{x}(t)=\frac{d x(t)}{d t}
$$

\section{Rolling Constraints: Unicycle-Like Robots}

As mentioned previously, mechanical systems that roll without slipping encompass the modeling for many mechanical systems (Bloch, 2015).

Moreover, according to Murray and Shankar (1993) any nonholonomic system can be written in a universal chain form, so unicycle models can be considered as a general nonholonomic dynamics.

In particular, unicycle-like robots represents a universal modeling for a wide variety of wheeled robots (Garcia and Agamennoni, 2012) and Fig. 1:

$\left[\begin{array}{c}\dot{x}(t) \\ \dot{y}(t) \\ \dot{\theta}(t)\end{array}\right]=\left[\begin{array}{cc}\cos (x) & 0 \\ \sin (x) & 0 \\ 0 & 1\end{array}\right] \cdot\left[\begin{array}{l}u_{1} \\ u_{2}\end{array}\right]$

where the control inputs $\left(u_{1}, u_{2}\right) \in \mathfrak{R}^{1} \times \mathfrak{R}^{1}$.

\section{Bessel's Functions Closed-form Solutions}

Following the ideas in Garcia et al. (2008), a lemma can be proved.

\section{Lemma 1}

Given the dynamics in Equation 1 driven by the controller:

$$
\begin{aligned}
& {\left[\begin{array}{l}
u_{1} \\
u_{2}
\end{array}\right]=\left[\begin{array}{c}
\sum_{i=1}^{N}(2 \cdot i+1) \cdot a \cdot C_{i} \cdot J_{i}(\theta) \cdot \theta^{i+1} \\
a \cdot \theta
\end{array}\right]} \\
& \theta(0)= \begin{cases}\theta(0), & \theta(0) \neq 0 \\
2 \cdot \pi, & \theta(0)=0\end{cases}
\end{aligned}
$$

For any arbitrary $N \in \mathbb{N}$, with $a<0$. The robot's trajectories are given by:

$$
\left[\begin{array}{c}
x(t) \\
y(t) \\
\theta(t)
\end{array}\right]=\left[\begin{array}{c}
R(\theta)^{\prime} \cdot \sum_{i=1}^{N} C_{i} \cdot\left[\begin{array}{c}
J_{i}(\theta) \cdot \theta^{i+1} \\
-J_{i+1}(\theta) \cdot \theta^{i+1}
\end{array}\right] \\
e^{a \cdot t} \cdot \theta(0)
\end{array}\right]
$$

With $J_{i}$ the Bessel's functions of first kind and $C_{i}$ arbitrary constants depending on the initial conditions. 


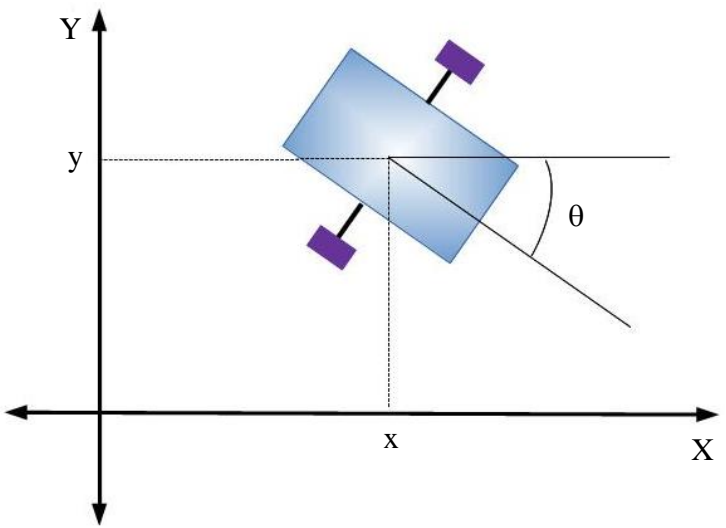

Fig. 1: Unicycle-like robot with coordinates

Proof

The proof is in the Appendix.

Equation 2 states clearly the origin as an equilibrium point if and only if $\lim _{t \rightarrow \infty} \theta(t)=0$.

\section{Unicycle's Asymptotic Stability}

Equation 1 defines a dynamics that it is endowed with uniform exponential stability by using Lemma 1 (Rugh (1995) for a definition on stability).

\section{Theorem 1}

The controller in Lemma 1 possess uniform exponentially stability to the origin.

\section{Proof}

The proof is in the Appendix.

Clearly, it is a closed-loop and time-invariant controller, except for $\theta(0)=0$, (rendering the controller identically zero).

However, the posture angle is a modular quantity:

$$
\theta(0)=0 \Leftrightarrow \theta(0)=2 \cdot \pi \bmod 2 \cdot \pi
$$

This modularity property avoids Brockett's condition collision. On the other hand, in a paper by Aicardi et al. (1995) a closed-loop controller using Lyapunov techniques was presented, however that controller becomes singular at $x(0)=0, y(0)=0$, whereas the controller in this paper is well defined for the whole $\mathfrak{R}^{3}$.

\section{Regular Embedded Sub-Manifold: Trajectories' First Integral}

Notwithstanding that the closed-form solution obtained represents the complete system's time evolution; a geometrical point of view is of interest in what follows.

Considering the closed-form trajectories in Lemma 1 with $N=2$ :

$$
\left[\begin{array}{l}
x(t) \\
y(t)
\end{array}\right]=R(\theta)^{\prime} \cdot\left(C_{1} \cdot\left[\begin{array}{c}
J_{1}(\theta) \cdot \theta^{2} \\
-J_{2}(\theta) \cdot \theta^{2}
\end{array}\right]+C_{2} \cdot\left[\begin{array}{c}
J_{2}(\theta) \cdot \theta^{3} \\
-J_{3}(\theta) \cdot \theta^{3}
\end{array}\right]\right)
$$

Compactly:

$$
\left[\begin{array}{l}
x(t) \\
y(t)
\end{array}\right]=R(\theta)^{\prime} \cdot L(\theta) \cdot \bar{C}
$$

Where:

$$
L(\theta)=\left[\begin{array}{cc}
J_{1}(\theta) \cdot \theta^{2} & J_{2}(\theta) \cdot \theta^{3} \\
-J_{2}(\theta) \cdot \theta^{2} & -J_{3}(\theta) \cdot \theta^{3}
\end{array}\right], \bar{C}=\left[\begin{array}{l}
C_{1} \\
C_{2}
\end{array}\right]
$$

Matrix $L(\theta)$ is nonsingular in the view of the Bourget's hypothesis proved in 1929 by Siegel (Watson, 1966).

Then, trajectories' regular embedded sub-manifold (first integral) follows (Bloch (2015), Isidori (1995) and Nijmeijer and van der Schaft (1990) for details in geometrical control):

$\bar{C}=L^{-1}(\theta) \cdot R(\theta) \cdot\left[\begin{array}{l}x(t) \\ y(t)\end{array}\right]$

Equivalenlty:

$$
L^{-1}(\theta) \cdot R(\theta) \cdot\left[\begin{array}{l}
x(t) \\
y(t)
\end{array}\right]=L^{-1}(\theta(0)) \cdot R(\theta(0)) \cdot\left[\begin{array}{l}
x(0) \\
y(0)
\end{array}\right]
$$

\section{Non-Linear Observer}

Equation 3 can be utilized to derive a non-linear observer measuring only the angle posture (Luenberger (1966) and Isidori (1995) for linear and non-linear observers):

$$
\begin{aligned}
& {\left[\begin{array}{l}
x(t) \\
y(t)
\end{array}\right]=R(\theta)^{\prime} \cdot L(\theta) \cdot L^{-1}(\theta(0)) .} \\
& \cdot R(\theta(0)) \cdot\left[\begin{array}{l}
x(0) \\
y(0)
\end{array}\right]
\end{aligned}
$$

\section{Practical Controller: Only Gyros}

Once that uniform exponential stability has been proved in Theorem 1, a practical algorithm can be described to control in closed-loop a unicycle robot:

- Determine the constant vector $\bar{C}$ from Equation 3 given the initial conditions 


$$
\bar{C}=\left[\begin{array}{l}
C_{1} \\
C_{2}
\end{array}\right]=L^{-1}(\theta(0)) \cdot R(\theta(0)) \cdot\left[\begin{array}{l}
x(0) \\
y(0)
\end{array}\right]
$$

- Use the controller in Lemma 1 with $N=2$ and single gyro measurements:

$$
\begin{aligned}
& {\left[\begin{array}{l}
u_{1} \\
u_{2}
\end{array}\right]=\left[\begin{array}{c}
a \cdot\left[3 \cdot C_{1} \cdot J_{1}(\theta) \cdot \theta^{2}+C_{2} \cdot J_{2}(\theta) \cdot \theta^{3}\right] \\
a \cdot \theta
\end{array}\right]} \\
& \theta(0)= \begin{cases}\theta(0), & \theta(0) \neq 0 \\
2 \cdot \pi, & \theta(0)=0\end{cases}
\end{aligned}
$$

Notice that only the initial condition must be provided to initiate the algorithm, endowing the controller with a very strong property for the well-known SLAM problem (Lavalle, 2006).

Only SLAM must be made at $t=0$ as opposed to the available literature where SLAM or time-tracking has to be performed on-line.

\section{Examples}

Using Matlab, Lemma 1 is implemented with both objectives: steering to the origin asymptotically stable and non-linear observer reconstruction.
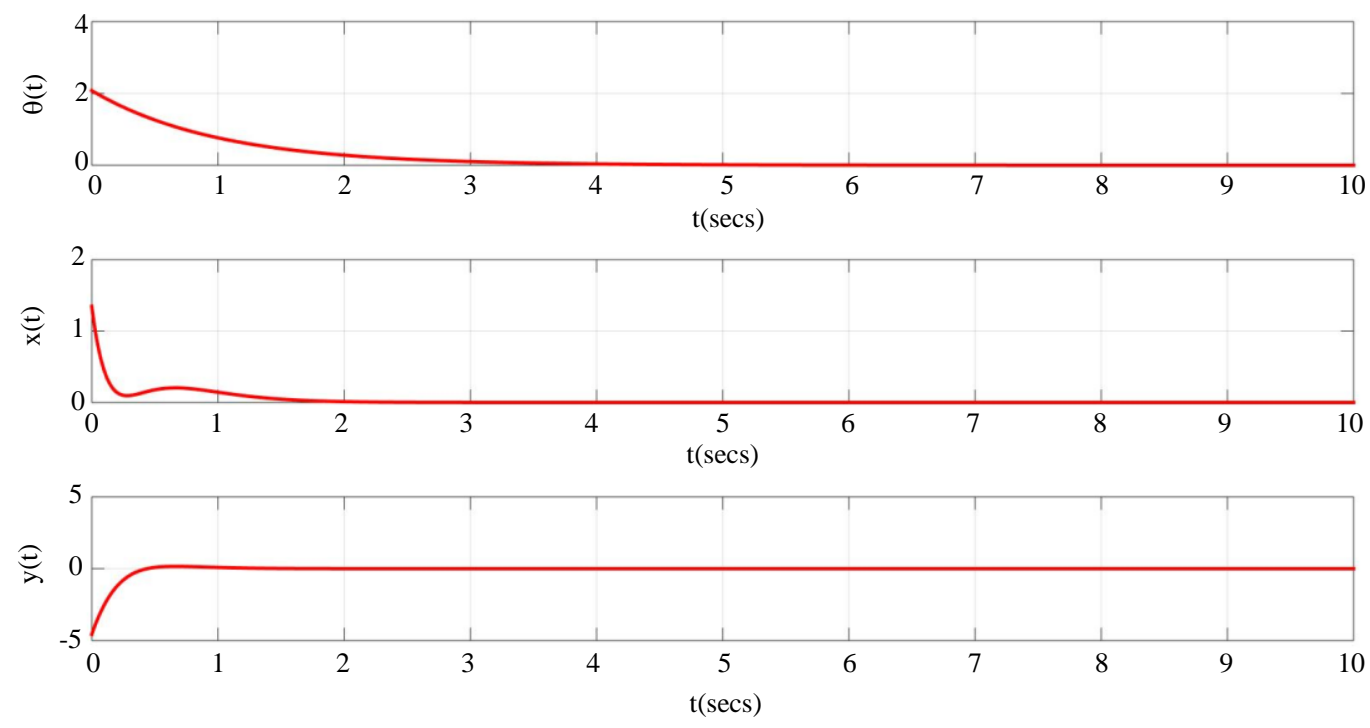

Fig. 2: Unicycle's closed-loop trajectories
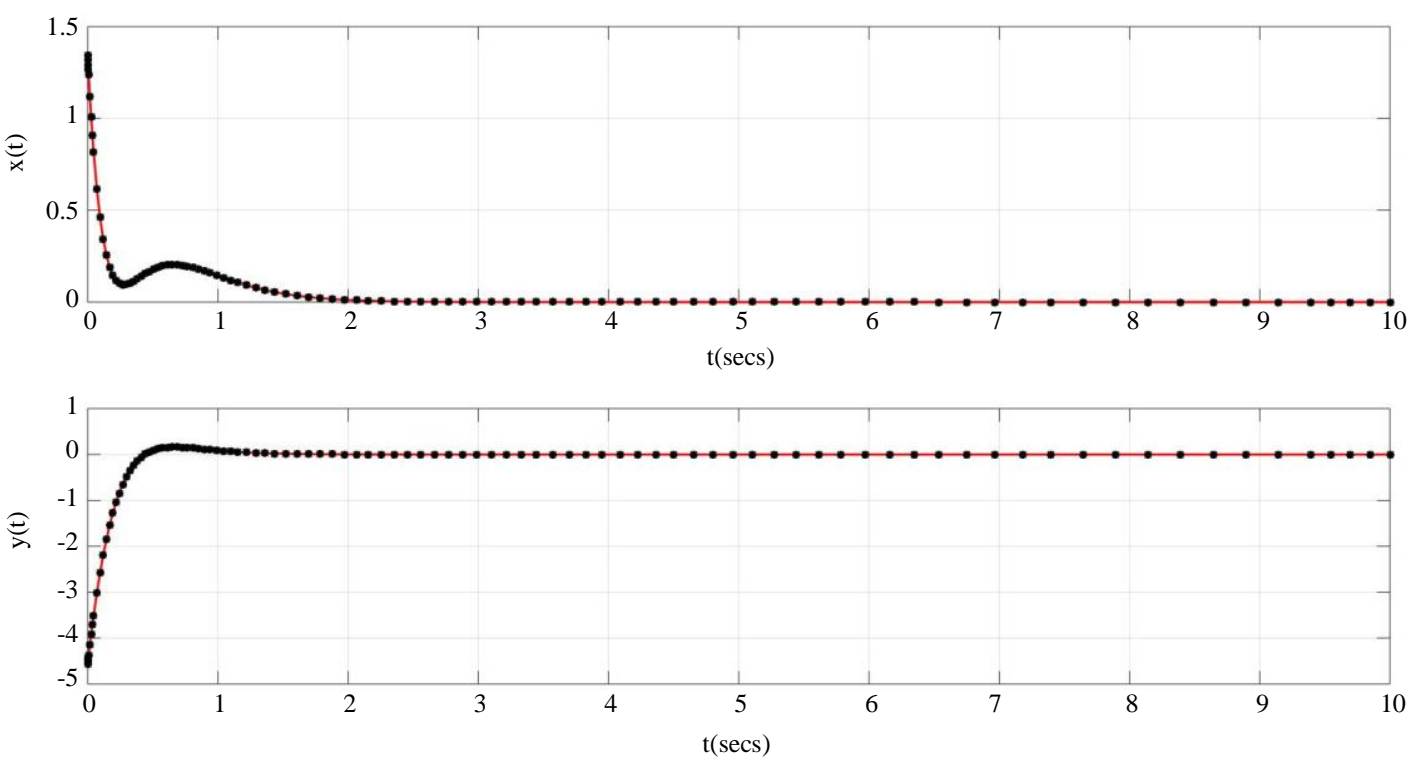

Fig. 3: Numerical verification 


\section{Using Gyros}

Considering the arbitrary initial condition: [1.345,4.567,2.078]'. Then, Fig. 2 is obtained.

\section{Non-Linear Observer Verification}

Equation 4 provides an interesting verification to numerically reconstruct robot's states. Then, Fig. 3 is obtained.

\section{Discussion}

Unicycle robot's asymptotic stability is not possible using a time-invariant feedback controller due to Brockett's condition.

In this paper, the modularity of the angular posture along with a novel pure feedback and time-invariant controller, allows asymptotic stability for unicycle robots.

It should be clear that modularity was not studied previously in the literature, excluding also closed form solutions addressed in this paper.

It turns out that, besides the fact of providing the important concept of a modular controller, SLAM and non-linear observers con be constructed in hardware using posture angle solely.

\section{Conclusion}

Nonholonomic trajectories of a unicycle-like robot is solved in closed-form using a smooth, closed-loop and time-invariant controller.

Uniform exponential stability was proved, even for this case (unicycle robot) where Brockett's condition is satisfied on the basis of modularity. In fact, postural angle's modularity was the cornerstone to avoid obstructions using smooth, closed-loop, time-invariant control laws.

Besides the wide variety of available literature, trajectories' closed-form knowledge allowing an explicit non-linear observer derivation to completely reconstruct states using only a single gyro sensor, makes a salient property of this paper.

Possible future work encompasses:

- Numerical algorithm to drive a set of multiple robots with additional constraints (formation of robots)

- Non-linear observer's numerical analysis robustness

- Real-time applications using on-board gyros and microprocessors

- $\quad$ Satellite control using gyros, applying the universal transformation in Murray and Shankar (1993)

- Optimal control

\section{Acknowledgement}

The author would like to acknowledge María de los Angeles, María de los Angeles and Alicia for their constant support.

\section{Funding Information}

This work is supported by Universidad Tecnológica Nacional-Facultad Regional Bahía Blanca under the project 5122TC.

\section{Ethics}

This article is original and contains unpublished material. The corresponding author confirms that all of the other authors have read and approved the manuscript and no ethical issues involved.

\section{References}

Andrews, L.C., 1985. Special Functions for Engineers and Applied Mathematics. 1st Edn., MacMillan Publishing Company, ISBN-10: 0070018448.

Angeles, J. and A. Kecskemethy, 1995. Kinematics and Dynamics of Multi-Body Systems. 1st Edn., Springer, New York, ISBN-10: 3211827315, pp: 342.

Aicardi, M., G. Casalino, A. Bicchi and A. Balestrino, 1995. Closed loop steering of unicycle like vehicles via Lyapunov Techniques. IEEE Robot. Automat. Magazine, 1: 27-35. DOI: 10.1109/100.388294

Astolfi, A., D.J.N. Limebeer, R.B. Vinter and Melchiorri, 1997. Modelling and Control of Mechanical Systems, Proceedings of the Workshop. 1st Edn., Wordl Scientific, ISBN-10: 1783262567, pp: 340 .

Bloch, A.M., 2015. Nonholonomic Mechanics and Control. 2nd Edn., Springer, New York, ISBN-10: 1493930168, pp: 565.

Brockett, R.W., 1983. Asymptotic stability and feedback stabilization. Proceedings of the Differential Geometric Control Theory, (GCT' 83), Birkhauser, pp: 181-191.

El-Hawwary, M.I. and M. Maggiore, 2008. Global path following for the unicycle and other results. Proceedings of the American Control Conference, Jun. 11-13, IEEE Xplore Press, Seattle, WA, USA, pp: 3500-3505. DOI: 10.1109/ACC.2008.4587035

Galceran, E. and M. Carreras, 2013. A survey on coverage path planning for robotics. Robot. Automat. Syst., 61: 1258-1276. DOI: $10.1016 /$ j.robot.2013.09.004

Garcia, A., O. Agamennoni and J. Figueroa, 2008. Closed-form solutions of the unicycle. Bahia Blanca-Argentina, arXiv: 0906.1588v1.

Garcia, A. and O. Agamennoni, 2012. Minimum-Time Control of Mobile Robots: Universal Modeling and Algorithms. 1st Edn., LAP LAMBERT Academic Publishing, ISBN-10: 3848412462, pp: 68.

Isidori, A., 1995. Nonlinear Control Systems. 3rd Ed. Springer, London, ISBN-10: 3540199160, pp: 549. 
Jian, Y., Q. Zhihua, W. Jing and K. Conrad, 2010. Comparison of optimal solutions to real-time path planning for a mobile vehicle. IEEE Trans. Syst. Man Cybernet., 4: 721-731. DOI: $10.1109 /$ TSMCA.2010.2044038

Kane, T.R. and D.A. Levinson, 1985. Dynamics, Theory and Applications. 1st Edn., MCGraw Hill Series in Mechancial Engineering, ISBN-10: 0070378460, pp: 379.

Kostić, D., S. Adinandra, J. Caarls, N. van de Wouw and H. Nijmeijer, 2009. Collision-free tracking control of unicycle mobile robots. Proceedings of the $48 \mathrm{~h}$ IEEE Conference on Decision and Control (CDC) held jointly with 2009 28th Chinese Control Conference, Dec. 15-18, IEEE Xplore Press, Shanghai, pp: 5667-5672. DOI: 10.1109/CDC.2009.5400088

Kumar, K. and A.K. Dewangan, 2016. Survey paper on robotics path planning algorithms. Int. J. Sci. Technol. Eng., 2: 1-4. DOI: 10.1155/2016/7426913

Lavalle, S.M., 2006. Planning Algorithms. 1st Ed. Cambridge University Press, New York, ISBN-13: 978-0521862059.

Li, Z.X. and J.F. Canny, 1992. Nonholonomic Motion Planning. 1st Edn., Kluwer, Dordrecht, The Netherlands, ISBN-13: 978-1-4613-6392-7.

Lizárraga, D., 2004. Obstructions to the existence of universal stabilizers for smooth control systems. Math. Control Signals Syst., 4: 255-277.

DOI: $10.1007 / \mathrm{s} 00498-003-0140-\mathrm{x}$

Luenberger, D., 1966. Observers for multivariable systems. IEEE Trans. Automatic Control, 2: 190-197.

DOI: 10.1109/TAC.1966.1098323

Moon Kim, B. and P. Tsiotras, 2002. Controllers for unicycle-type wheeled robots: Theoretical results and experimental validation. IEEE Trans. Robot. Automat., 3: 294-307. DOI: 10.1109/TRA.2002.1019460

Morro, A., A. Sgorbissa and R. Zaccaria, 2011. Path following for unycicle robots with arbitrary path curvatures. IEEE Trans. Robot., 5: 1016-1023. DOI: $10.1109 /$ TRO.2011.2148250

Muñoz-Lecanda, M.C. and F.J. Yániz Fernandez, 2008. Mechanical control systems and kinematics. IEEE Trans. Automatic Control, 5: 1297-1302. DOI: 10.1109/TAC.2008.921004

Murray, R. and S. Shankar, 1993. Nonholonomic motion planning: Steering using sinusoids. IEEE Trans. Automatic Control, 5: 700-716.

DOI: 10.1109/9.277235

Nijmeijer, H. and A.J. van der Schaft, 1990. Nonlinear Dynamical Control Systems. 2nd Edn., SpringerVerlag, ISBN-10: 038797234X, pp: 467.

Qu, Z., J. Wang and C.E. Plaisted, 2004. A new analytical solutions to mobile robot trajectory generation in the presence of moving obstacles. IEEE Trans. Robot., 6: 978-993.

DOI: $10.1109 /$ TRO.2004.829461
Rugh, W., 1995. Linear System Theory. 2nd Edn., Pearson, ISBN-10: 0134412052.

Salaris, P., D. Fontanelli, L. Pallottino and A. Bicchi, 2010. Shortest paths for a robot with nonholonomic and field-of-view constraints. IEEE Trans. Robot., 2: 269-281. DOI: 10.1109/TRO.2009.2039379

Sarkar, N., X. Yun and V. Kumar, 1994. Control of mechanical systems with rolling constraints application to dynamic control of mobile. Int. J. Robot. Res., 1: 1-27. DOI: $10.1177 / 027836499401300104$

Siegwart, R., I.R. Nourbakhsh and D. Scaramuzza, 2011. Introduction to Autonomous Mobile Robots. 2nd Edn., The MIT Press, ISBN-10: 0262015358, pp: 472.

Skowronski, J.M., 2012. Control of Nonlinear Mechanical Systems. 1st Edn., Springer, ISBN-13: 978-0306438271.

Thomas, M., B. Bandyopadhyay and L. Vachhani, 2016. Posture stabilization of unicycle mobile robot using finite time control techniques. IFAC-PapersOnLine, 49: 379-384. DOI: 10.1016/j.ifacol.2016.03.083

Udwadia, F.E. and R.E. Kalaba, 1994. A new perspective on constrained motion. Proc. Royal Society London, 439: 407-410. DOI: $10.1007 / 978-1-4615-2425-0 \_8$

Wang, H., Y. Chen and P. Souéres, 2009. A geometric algorithm to compute time-optimal trajectories for a bidirectional steered robot. IEEE Trans. Robot., 2: 399-413. DOI: 10.1109/TRO.2009.2015610

Watson, G., 1966. A Treatise on the Theory of Bessel Functions. 2nd Edn., Cambridge University Press, ISBN-13: 978-0521483919.

Yang, L., J. Qi, D. Song, J. Xiao and J. Han et al., 2016. Survey of robot 3D path planning algorithms. J. Control Sci. Eng. DOI: 10.1155/2016/7426913

Zambelli, M., Y. Karayiannidis and D. Dimarogonas, 2015. Posture regulation for unicycle-like robots with prescribed performance guarantees. IET Control Theory Applic., 1: 192-202.

DOI: $10.1049 /$ iet-cta.2013.1112

\section{Appendix}

\section{Lemma 1 Proof}

Let's consider an auxiliary system:

$$
\dot{X}_{i}=\left[\begin{array}{c}
\cos (\theta) \\
\sin (\theta)
\end{array}\right] \cdot u_{i}, \quad i=1, \cdots, N
$$

Summing up:

$$
\sum_{i=1}^{N} \dot{X}_{i}=\left[\begin{array}{c}
\cos (\theta) \\
\sin (\theta)
\end{array}\right] \cdot \sum_{i=1}^{N} u_{i}
$$


Defining:

$$
X=\sum_{i=1}^{N} X_{i} \Rightarrow u_{1}=\sum_{i=1}^{N} u_{i}
$$

Considering the inspiring guess:

$$
\begin{aligned}
& u_{i}=a \cdot(2 \cdot i+1) \cdot\left[\begin{array}{ll}
\cos (\theta) & \sin (\theta)
\end{array}\right] \cdot X_{i} \\
& a<0, \quad i=1, \cdots, N
\end{aligned}
$$

Then:

$$
\dot{X}_{i}=a \cdot(2 \cdot i+1) \cdot R(\theta)^{\prime} \cdot\left[\begin{array}{l}
1 \\
0
\end{array}\right] \cdot\left[\begin{array}{ll}
1 & 0
\end{array}\right] \cdot R(\theta)
$$

Defining the change of coordinates:

$$
Z_{i}=R(\theta) \cdot X_{i}
$$

Taking derivatives with respect to time:

$$
\dot{Z}_{i}=\left[\begin{array}{cc}
(2 \cdot i+1) \cdot a & \dot{\theta} \\
-\dot{\theta} & 0
\end{array}\right] \cdot Z_{i}
$$

Defining the control input:

$$
\begin{aligned}
& u_{2}=a \cdot \theta, \quad a<0 \\
& \theta(0)= \begin{cases}\theta(0), & \theta(0) \neq 0 \\
2 \cdot \pi, & \theta(0)=0\end{cases}
\end{aligned}
$$

Equation 6 leads:

$$
\theta \cdot \frac{d Z_{i}}{d \theta}=\left[\begin{array}{cc}
(2 \cdot i+1) & \theta \\
-\theta & 0
\end{array}\right] \cdot Z_{i}
$$

Equation 5 means:

$$
u_{i}=a \cdot(2 \cdot i+1) \cdot\left[\begin{array}{ll}
1 & 0
\end{array}\right] \cdot Z_{i}
$$

So, only the first component of vector $Z_{i}$ needs to be considered. Taking derivative with respect to $\theta$ in Equation 7:

$$
\theta^{2} \cdot \frac{d Z_{i 1}(\theta)}{d \theta}=\theta \cdot(2 \cdot i+1) \cdot \frac{d Z_{i 1}(\theta)}{d \theta}+\left(2 \cdot i+1+\theta^{2}\right) \cdot Z_{i 1}(\theta)
$$

For this Bessel's ODE, a closed-form solution is known (Andrews (1985) pp.229):

$$
Z_{i 1}(\theta)=\theta^{i+1} \cdot\left(C_{i} \cdot J_{i}(\theta)+D_{i} \cdot Y_{i}(\theta)\right)
$$

With $\left\{J_{i}, Y_{i}\right\}$ the Bessel's functions of first and second kind respectively. Assuming $D_{i}=0$. Finally, for $Z_{i 2}$ from Equation (6):

$$
\left\{\begin{array}{l}
Z_{i 2}(\theta)=\frac{d Z_{i 1}(\theta)}{d \theta}-\frac{(2 \cdot i+1)}{\theta} \cdot Z_{i 1}(\theta) \\
Z_{i 1}(\theta)=\theta^{i+1} \cdot C_{i} \cdot J_{i}(\theta)
\end{array}\right.
$$

Utilizing the Bessel's function property:

$$
\frac{d J_{i}(\theta)}{d \theta}=\frac{i}{\theta} \cdot J_{i}(\theta)-J_{i+1}(\theta)
$$

Then:

$$
\begin{aligned}
& Z_{i 2}(\theta)=C_{i} \cdot\left[\frac{i}{\theta} \cdot J_{i}(\theta) \cdot \theta^{i+1}-J_{i}(\theta) \cdot \theta^{i+1}\right. \\
& \left.+(i+1) \cdot J_{i}(\theta) \cdot \theta^{i}+-\frac{(2 i+1)}{\theta} \cdot J_{i}(\theta) \cdot \theta^{i+1}\right]
\end{aligned}
$$

Equivalently:

$$
Z_{i 2}(\theta)=C_{i} \cdot\left[-\theta^{i+1} \cdot J_{i+1}(\theta)\right]
$$

This completes the proof.

\section{Theorem 1 Proof}

First, the origin must be an equilibrium point. Equation 2 shows that the origin is an equilibrium point as long as $\theta(t)$ is exponentially tending to zero.

Moreover, this equilibrium point is attractive to the origin uniformly (taking into account the Bessel's functions of first kind's uniform decay behavior):

$$
\dot{\theta}(t)=a \cdot \theta(t), \quad a<0 \Rightarrow \lim _{t \rightarrow \infty} \theta(t)=0 \text { (Uniformly) }
$$$$
\left[\begin{array}{l}
x(t) \\
y(t)
\end{array}\right]=R(\theta) \cdot \sum_{i=1}^{N} C_{i} \cdot \theta^{i+1} \cdot\left[\begin{array}{c}
J_{i}(\theta) \\
-J_{i+1}(\theta)
\end{array}\right] \rightarrow 0 \text { (Uniformly) }
$$

Finally, an exponential bound is proved:

$$
\left\|\left[\begin{array}{c}
x(t) \\
y(t)
\end{array}\right]\right\| \leq\|R(\theta)\| \cdot \sum_{i=1}^{N} C_{i} \cdot\left|\theta^{i+1}\right| \cdot\left\|\left[\begin{array}{c}
J_{i}(\theta) \\
-J_{i+1}(\theta)
\end{array}\right]\right\|
$$

Bessel's functions are bounded at the origin $(\theta=0)$, so uniform exponentially stability is proved. This completes the proof. 